\title{
Cardinal Interpolation with Polysplines on Annuli
}

\author{
O. Kounchev ${ }^{1}$ \\ Institute of Mathematics and Informatics, Bulgarian Academy of Sciences, 8 \\ Acad. G. Bonchev Str., 1113 Sofia, Bulgaria \\ H. Render ${ }^{2}$ \\ Departamento de Matemáticas y Computación, Universidad de La Rioja, Edificio \\ Vives, Luis de Ulloa s/n., 26004 Logroño, Spain
}

\begin{abstract}
Cardinal polysplines of order $p$ on annuli are functions in $C^{2 p-2}\left(\mathbb{R}^{n} \backslash\{0\}\right)$ which are piecewise polyharmonic of order $p$ such that $\Delta^{p-1} S$ may have discontinuities on spheres in $\mathbb{R}^{n}$, centered at the origin and having radii of the form $e^{j}, j \in \mathbb{Z}$. The main result is an interpolation theorem for cardinal polysplines where the data are given by sufficiently smooth functions on the spheres of radius $e^{j}$ and center 0 obeying a certain growth condition in $|j|$. This result can be considered as an analogue of the famous interpolation theorem of Schoenberg for cardinal splines.
\end{abstract}

Key words: Cardinal splines, Schoenberg interpolation theorems, $L$-splines, cardinal spline interpolation, spherical harmonics, polyharmonic functions in annulus, biharmonic functions, polysplines.

1991 MSC: MSC Code Primary 41A15, Secondary 35J40, 31B30.

Email addresses: kounchev@math.bas.bg (O. Kounchev), render@gmx.de (H. Render).

1 Partially sponsored by the Fulbright Program during his stay at the University of Wisconsin-Madison.

2 Supported by the Alexander von Humboldt-Foundation in the framework of the Feodor Lynen Program. 


\section{Introduction}

Polysplines have been introduced by the first author as a multivariate analog of splines in one variable, see e.g. [9]. In the monograph [10] applications of polysplines to Multiresolutional Analysis and Wavelet Analysis in the spirit of the work of Chui (see [5]) have been given. In this paper an interpolation result for cardinal polysplines on annuli (defined below) will be presented which is motivated by the work of I. Schoenberg on cardinal spline interpolation, see [19].

Let $p$ and $n$ be natural numbers which are fixed throughout the paper and let $\mathbb{R}^{n}$ be the $n$-dimenisonal euclidean space and $\mathbb{Z}$ the set of all integers. As in [11],[12],[13] a function $S: \mathbb{R}^{n} \backslash\{0\} \rightarrow \mathbb{C}$ is called a cardinal polyspline of order $p$ on annuli if $S$ is $(2 p-2)$-times continuously differentiable and the restriction of $S$ to each open annulus

$$
A_{j}:=\left\{x \in \mathbb{R}^{n}: e^{j}<|x|<e^{j+1}\right\}
$$

is a polyharmonic function of order $p$ for $j \in \mathbb{Z}$. Recall that a function $f$ defined on an open set $U$ in $\mathbb{R}^{n}$ is polyharmonic of order $p$ if $f$ is $2 p$-times continuously differentiable and $\Delta^{p} f(x)=0$ for all $x \in U$ where $\Delta$ is the Laplace operator and $\Delta^{p}$ its $p$-th iterate. It is well known that a polyharmonic function is real analytic, hence infinitely differentiable. Hence after differentiating a polyspline $(2 p-2)$ times one may have discontinuities only on the spheres $e^{j} \mathbb{S}^{n-1}=$ $\left\{e^{j} y: y \in \mathbb{S}^{n-1}\right\}$ with $j \in \mathbb{Z}$, where

$$
\mathbb{S}^{n-1}=\left\{y \in \mathbb{R}^{n}:|y|=1\right\}
$$

is the unit sphere. So one may see the spheres $e^{j} \mathbb{S}^{n-1}, j \in \mathbb{Z}$, as the multivariate analog of the notion of the knots $j \in \mathbb{Z}$ of a cardinal spline in the univariate case. Later it will become clear why these radii are of the form $e^{j}, j \in \mathbb{Z}$.

Schoenberg's famous interpolation theorem for cardinal splines of odd degree says that for data given on the knots $j \in \mathbb{Z}$ of polynomial growth in $j \in \mathbb{Z}$ there exists a cardinal spline interpolating the data which is of the same polynomial growth on the real line, see [19, p. 34]. The aim of this paper is to present an analog of Schoenberg's result for polysplines in the following way: the data are given by functions $d_{j}: e^{j} \mathbb{S}^{n-1} \rightarrow \mathbb{C}$ for $j \in \mathbb{Z}$ and we want to find a polyspline $S: \mathbb{R}^{n} \backslash\{0\} \rightarrow \mathbb{C}$ which interpolates the data, i.e. that

$$
S(y)=d_{j}(y) \text { for all } y \in e^{j} \mathbb{S}^{n-1} \text { and } j \in \mathbb{Z}
$$

and which has a similar growth as the data. Clearly we have to assume that 
the data functions $d_{j}$ are at least $(2 p-2)$ times continuously differentiable. It turns out that the results are naturally formulated in the context of the Sobolev spaces $H^{s, 1}\left(\mathbb{S}^{n-1}\right)$ for appropriate $s>0$, for details see Section 6 .

Our main result states the following: Let $\gamma \geq 0$ be fixed; for $s=s_{p, n}=$ $2(p-1)+(n / 2)-1$ and $f_{j} \in H^{s, 1}\left(\mathbb{S}^{n-1}\right), j \in \mathbb{Z}$, define functions $d_{j}: e^{j} \mathbb{S}^{n-1} \rightarrow$ $\mathbb{C}$ by $d_{j}\left(e^{j} \theta\right)=f_{j}(\theta)$ for $\theta \in \mathbb{S}^{n-1}$. Assume that the data functions obey the growth condition

$$
\left\|f_{j}\right\|_{s} \leq C\left|\log e^{j}\right|^{\gamma} \text { for all } j \in \mathbb{Z}
$$

Then there exists a polyspline $S$ of order $p$ interpolating the data functions $d_{j}$ (i.e. (1)) and satisfying the estimate

$$
|S(x)| \leq D|\log | x||^{\gamma} \text { for all } x \in \mathbb{R}^{n} .
$$

In order to explain the construction of $S$ recall that a function $u: \mathbb{R} \rightarrow$ $\mathbb{R}$ is a cardinal $L$-spline (here $L$ stands for a linear differential operator with constant coefficients of degree $N+1)$ if $u$ is $(N-1)$-times continuously differentiable and if for every $l \in \mathbb{Z}$ there exists an infinitely differentiable function $f_{l}: \mathbb{R} \rightarrow \mathbb{C}$ with $L f=0$ such that $u(t)=f_{l}(t)$ for all $t \in(l,(l+1))$. The essence of our construction involves writing the Laplacian in spherical coordinates, expanding the polyspline $S$ in a series of spherical harmonics, and, using the Michelli theory of cardinal $L$-splines, glueing the radial part together to get $S$; roughly speaking, this means that a polyspline can be written in the form

$$
S(x)=\sum_{k=0}^{\infty} \sum_{l=1}^{a_{k}} S_{k, l}(\log |x|) Y_{k, l}\left(\frac{x}{|x|}\right)
$$

where $Y_{k, l}, k=0,1, \ldots, l=1, \ldots, a_{k}$, is a basis for the set of all spherical harmonics and the coefficients $S_{k, l}$ are $L$-splines with respect to the linear differential operator $M_{\Lambda(k)}$ defined in (3). In order to achieve convergence of the sum one needs precise estimates for the fundamental $L$-splines taking into account their dependence on the parameter $k$.

The paper is structured as follows: Section 2 gives some basic facts about polysplines and spherical harmonics in order to clarify the connection between polysplines and $L$-splines. In Section 3 we give a brief account of the theory of Charles Micchelli who has generalized in [16], [17] the results of Schoenberg on polynomial splines to the setting of $L$-splines.

In Section 4 we discuss asymptotic estimates of the Euler-Frobenius function (defined in Section 3) depending on the parameter $k \in \mathbb{N}_{0}$. In Section 5 we 
use these asymptotics to obtain uniform estimates of fundamental $L$-splines containing the parameter $k$. Section 6 contains our main result. Uniqueness of the interpolation splines will be shown in the last section.

\section{Spherical harmonics and polysplines}

Each $x \in \mathbb{R}^{n}$ will be written in spherical coordinates $x=r \theta$ with $r \geq 0$ and $\theta \in \mathbb{S}^{n-1}:=\left\{x \in \mathbb{R}^{n}:|x|=1\right\}$. Recall that a function $Y: \mathbb{S}^{n-1} \rightarrow \mathbb{C}$ is a spherical harmonic of degree $k \in \mathbb{N}_{0}$ if there exists a homogeneous harmonic polynomial $P(x)$ of degree $k$ such that $P(\theta)=Y(\theta)$ for all $\theta \in \mathbb{S}^{n-1}$. By $a_{k}$ we will denote the dimension of the vector space $\mathcal{H}_{k}$ of all spherical harmonics of degree exactly $k$. By $Y_{k, l}(\theta), l=1, \ldots, a_{k}$ we will denote an orthonormal basis of the space $\mathcal{H}_{k}$ endowed with the scalar product

$$
\int_{\mathbb{S}^{n-1}} f(\theta) \overline{g(\theta)} d \theta \text {. }
$$

For the reader not familiar with spherical harmonics, it might be useful to consider the two-dimensional case: identify $\mathbb{S}^{1}$ with $[0,2 \pi)$ and choose as a basis $Y_{0}=\frac{1}{\sqrt{2 \pi}}$ and

$$
Y_{k, 1}(t)=\frac{1}{\sqrt{\pi}} \cos k t \text { and } Y_{k, 2}(t)=\frac{1}{\sqrt{\pi}} \sin k t .
$$

For a detailed account we refer to [23] or [2].

Let $R_{1}<R_{2}$ be positive real numbers and let $\left(R_{1}, R_{2}\right)$ be the open interval $\left\{r \in \mathbb{R}: R_{1}<r<R_{2}\right\}$. Assume that $u:\left(R_{1}, R_{2}\right) \rightarrow \mathbb{C}$ be infinitely differentiable and $Y_{k} \in \mathcal{H}_{k}$. Then it is well known (see e.g. [10, p. 152]) that $\Delta\left(u(r) Y_{k}(\theta)\right)=Y_{k}(\theta) L_{(k)} u(r)$ where

$$
L_{(k)}=\frac{d^{2}}{d r^{2}}+\frac{n-1}{r} \frac{d}{d r}-\frac{k(k+n-2)}{r^{2}} .
$$

By iteration we have $\Delta^{p} u=Y_{k}(\theta) \cdot\left[L_{(k)}\right]^{p} u(r)$. Thus the function $u(r, \theta)=$ $u(r) Y_{k}(\theta)$ is polyharmonic of order $p$ if and only if $\left[L_{(k)}\right]^{p} u(r)=0$ for all $r \in\left(R_{1}, R_{2}\right)$.

Let us put for convenience

$$
\Lambda_{+}(k) \quad:=\{k, k+2, \ldots, k+2 p-2\},
$$




$$
\Lambda_{-}(k):=\{-k-n+2,-k-n+4, \ldots,-k-n+2 p\} .
$$

The space of solutions of the equation $L_{(k)}^{p} f(r)=0$ which are $C^{\infty}$ for $r>0$ is generated by a simple basis: for $j \in \Lambda_{+}(k) \cup \Lambda_{-}(k)$ the function $r^{j}$ is clearly a solution, while for $j \in \Lambda_{+}(k) \cap \Lambda_{-}(k)$ we obtain a second solution $r^{j} \log r$. It will be convenient to make a transform $v=\log r$. Then a solution of the form $r^{j}$ will be transformed to $e^{j v}$ and a solution of the form $r^{j} \log r$ is transformed to $v e^{j v}$. We see immediately that all solutions to the equation $L_{(k)}^{p} f(r)=0$ are transformed to solutions of the equation $M_{\Lambda(k)} g(v)=0$ where $M_{\Lambda(k)}$ is the constant coefficient linear differential operator defined by

$$
M_{\Lambda(k)}:=\prod_{\lambda \in \Lambda_{+}(k)}\left(\frac{d}{d v}-\lambda\right) \prod_{\lambda \in \Lambda_{-}(k)}\left(\frac{d}{d v}-\lambda\right) .
$$

Later we shall also use the notation

$$
\Lambda(k)=(k, \ldots, k+2 p-2,-k-n+2, \ldots,-k-n+2 p)
$$

which is a vector taking all values from $\Lambda_{+}(k)$ and $\Lambda_{-}(k)$ (including multiplicities). From this we have immediately

Proposition 1 Let $N$ be a natural number and suppose that $S_{k, l}: \mathbb{R} \rightarrow \mathbb{C}$ are cardinal L-splines with respect to the differential operator $M_{\Lambda(k)}$ for $k=$ $0, \ldots, N, l=1, \ldots, a_{k}$. Then the function $S: \mathbb{R}^{n} \backslash\{0\} \rightarrow \mathbb{C}$ defined for $x=r \theta$ with $r>0$ and $\theta \in \mathbb{S}^{n-1}$ by

$$
S(r \theta)=\sum_{k=0}^{N} \sum_{l=1}^{a_{k}} S_{k, l}(\log r) Y_{k, l}(\theta)
$$

is a cardinal polyspline of order $p$.

It might be a temptation to say that cardinal polysplines are just the functions of the form

$$
S(r \theta)=\sum_{k=0}^{\infty} \sum_{l=1}^{a_{k}} S_{k, l}(\log r) Y_{k, l}(\theta)
$$

where $S_{k, l}$ are $L$-splines with respect to $M_{\Lambda(k)}$; however, one has to be careful since the convergence of the sum has to be justified and the differentiability of the function $S$ defined in (5) up to the order $2 p-2$ is not a consequence of the absolute convergence of the sum. 
On the other hand, we mention the following result in [12] which will be used in the last Section to prove uniqueness of interpolation with polysplines.

Theorem 2 Let $S: \mathbb{R}^{n} \backslash\{0\} \rightarrow \mathbb{C}$ be a cardinal polyspline of order $p$. Then the function $S_{k, l}: \mathbb{R} \rightarrow \mathbb{C}$ defined by

$$
S_{k, l}(v):=\int_{\mathbb{S}^{n-1}} S\left(e^{v} \theta\right) Y_{k, l}(\theta) d \theta
$$

is a cardinal $L$-spline with respect to $M_{\Lambda(k)}$ for $k \in \mathbb{N}_{0}, l=1, \ldots, a_{k}$.

\section{Cardinal $L-$ splines}

The previous Section has shown that polysplines are intimately related to a sequence of $L$-splines given by the Fourier coefficients of the polysplines.

Micchelli has worked out in [16], [17] a theory of cardinal $L$-splines with respect to a linear differential operator $L$ (of order $N+1$ ) with constant coefficients. As in [16] $\Lambda:=\left(\lambda_{1}, \lambda_{2}, \ldots, \lambda_{N+1}\right)$ denotes an (unordered) vector with repetitions according to the multiplicities with real coefficients $\lambda_{j}, j=$ $1, \ldots, N+1$. Then $L$ defined by

$$
L:=\prod_{j=1}^{N+1}\left(\frac{d}{d x}-\lambda_{j}\right)
$$

is a linear differential operator of order $N+1$. Let us define the polynomial $q_{\Lambda}$ as

$$
q_{\Lambda}(z):=\prod_{j=1}^{N+1}\left(z-\lambda_{j}\right)
$$

and $e^{\Lambda}=\left\{e^{\lambda_{j}}: j=1, \ldots, N+1\right\}$. In the theory of cardinal $L$-splines the function $A_{\Lambda}: \mathbb{R} \times\left(\mathbb{C} \backslash e^{\Lambda}\right) \rightarrow \mathbb{C}($ cf. [17], p. 223) defined by

$$
A_{\Lambda}(x, \lambda)=\frac{1}{2 \pi i} \int_{\Gamma} \frac{1}{q_{\Lambda}(z)} \frac{e^{x z}}{e^{z}-\lambda} d z
$$

is of fundamental importance. Here $\Gamma$ is a closed simple curve in the complex plane surrounding all $\lambda_{j}, j=1, \ldots, N+1$ and having the zeros of the function 
$e^{z}-\lambda$ in the exterior of $\Gamma$. The Euler-Frobenius function is defined by

$$
\Pi_{\Lambda}(x, \lambda):=A_{\Lambda}(x, \lambda) \cdot \prod_{j=1}^{N+1}\left(e^{\lambda_{j}}-\lambda\right) .
$$

For $x=0$ it is a polynomial of degree at most $N$ in the variable $\lambda$ (Corollary 2.1 in [17]) and $\Pi_{\Lambda}(0, \lambda)$ is called the Euler-Frobenius polynomial. Next we recall the definition of the so-called basis spline which will be denoted by $Q_{\Lambda}$ : Define the function $s_{\Lambda}(\lambda):=\prod_{j=1}^{N+1}\left(e^{-\lambda_{j}}-\lambda\right)$ and let $s_{j}, j=0, \ldots, N+1$ be the coefficients of $s_{\Lambda}(\lambda)$, i.e. $s_{\Lambda}(\lambda)=\sum_{j=0}^{N+1} s_{j} \lambda^{j}$. Due to the choice of the real number $s_{j}$ it is straightforward to prove that the following cardinal $L$-spline has support in the interval $[0, N+1]$, namely

$$
Q_{\Lambda}(x):=\sum_{j=0}^{N+1} s_{j} \cdot A_{\Lambda}(x-j, 0) \cdot 1_{[0, \infty)}(x) .
$$

The following fundamental formula relates the Euler-Frobenius function with the basis-spline (cf. [17, p. 221 and p. 222]) for $0 \leq x \leq 1$,

$$
R_{\Lambda}^{x}(\lambda):=\sum_{j=0}^{N} \lambda^{N-j} Q_{\Lambda}(x+j)=\frac{(-1)^{N}}{e^{\left(\lambda_{1}+\ldots+\lambda_{N+1}\right)}} \cdot \Pi_{\Lambda}(x, \lambda) .
$$

\subsection{The fundamental $L-$ spline}

Let us now consider the interpolation problem for cardinal $L-$ splines. A cardinal $L$-spline $L_{\Lambda}$ is called fundamental $L$-spline if $L_{\Lambda}(0)=1$ and $L_{\Lambda}(j)=0$ for all $j \in \mathbb{Z}, j \neq 0$ and if it decays exponentially, i.e. if there exist two constants $A, B>0$ such that

$$
\left|L_{\Lambda}(x)\right| \leq A e^{-B|x|} \text { for all } x \in \mathbb{R} .
$$

We cite the following result from [17, Corollary 2.3] :

Theorem 3 If $A_{\Lambda}(0,-1) \neq 0$ then there exists a unique fundamental $L$ spline.

We now recall from $\left[20\right.$, p. 271] the construction of the fundamental spline $L_{\Lambda}$ since we need a detailed knowledge of the constants $A$ and $B$ in the estimate (12). Define

$$
P_{\Lambda}(\lambda):=R_{\Lambda}^{0}\left(\frac{1}{\lambda}\right) \lambda^{N}=\sum_{j=0}^{N} \lambda^{j} Q_{\Lambda}(j) .
$$


The following result in [17, Corollary 2.3] shows that $P_{\Lambda}$ has no zeros on the unit circle:

Proposition 4 The function $1 / P_{\Lambda}(\lambda)$ is holomorphic in a neighborhood of the unit circle if and only if $A_{\Lambda}(0,-1) \neq 0$.

Assume now that the function $\lambda \rightarrow 1 / P_{\Lambda}(\lambda)$ is holomorphic on the annulus $\left\{R_{1}<|\lambda|<R_{2}\right\}$ (where $R_{1}<1<R_{2}$ ), and consider its Laurent series

$$
\frac{1}{P_{\Lambda}(\lambda)}=\sum_{j=-\infty}^{\infty} \omega_{j} \lambda^{j}
$$

According to $\left[20\right.$, p. 271] the fundamental $L$-spline $L_{\Lambda}$ is given by

$$
L_{\Lambda}(x):=\sum_{j=-\infty}^{\infty} \omega_{j} Q_{\Lambda}(x-j) \text {. }
$$

The series in (14) converges absolutely and locally uniformly. The estimate in the next proposition is straightforward using the Cauchy estimates for the coefficients of a Laurent series. The somewhat technical proof is omitted.

Proposition 5 Let $\Lambda=\left(\lambda_{1}, \ldots, \lambda_{N+1}\right)$. Suppose that $1 / P_{\Lambda}(\lambda)$ is holomorphic on the annulus $\left\{R_{1}<|\lambda|<R_{2}\right\}$ with $R_{1}<1<R_{2}$. Let $\rho>0$ with $R_{1}<$ $\rho<1<\frac{1}{\rho}<R_{2}$ and put $\varepsilon=-\log \rho>0$. Then there exists a constant $G(\rho)$ depending only on $\rho$ and $N$ such that

$$
\left|L_{\Lambda}(x)\right| \leq G(\rho) \max _{y \in(0, N+1)}\left|Q_{\Lambda}(y)\right| \cdot \max _{\rho \leq|\lambda| \leq 1 / \rho} \frac{1}{\left|P_{\Lambda}(\lambda)\right|} \cdot e^{-\varepsilon|x|} .
$$

We mention that the same proof yields the inequality

$$
\left|\frac{d^{m}}{d x^{m}} L_{\Lambda}(x)\right| \leq G(\rho) \max _{y \in(0, N+1)}\left|\frac{d^{m}}{d y^{m}} Q_{\Lambda}(y)\right| \cdot \max _{\rho \leq|\lambda| \leq 1 / \rho} \frac{1}{\left|P_{\Lambda}(\lambda)\right|} \cdot e^{-\varepsilon|x|}
$$

for each $m=0, \ldots, N-1$.

\subsection{Estimate of $\max Q_{\Lambda}$}

In the following we want to give an estimate of the basis spline $Q_{\Lambda}$ and its derivatives, i.e. we want to estimate $\left|\frac{d^{m}}{d x^{m}} Q_{\Lambda}(x)\right|$ where $m$ satisfies $0 \leq m \leq$ $N-1$. For this we define for given $\Lambda=\left(\lambda_{1}, \ldots, \lambda_{N+1}\right)$ the number

$$
M_{\Lambda}:=\max \left\{\left|\lambda_{1}\right|, \ldots,\left|\lambda_{N+1}\right|\right\}
$$


and for $M_{\Lambda} \neq 0$ we put

$$
B_{\Lambda}(m):=\sum_{k=0}^{m} M_{\Lambda}^{-k} \max _{0 \leq x \leq 1}\left|A_{\left(\lambda_{1}, \ldots, \lambda_{N+1-k}\right)}(x, 1)\right| .
$$

Note that $B_{\Lambda}(0)=\max _{0 \leq x \leq 1}\left|A_{\left(\lambda_{1}, \ldots, \lambda_{N+1}\right)}(x, 1)\right|$ and $B_{\Lambda}(m) \leq B_{\Lambda}(m+1)$.

Recall that $r_{\Lambda}(\lambda)=\prod_{j=1}^{N+1}\left(e^{\lambda_{j}}-\lambda\right)$.

Theorem 6 Let $N \in \mathbb{N}_{0}$ and $\delta>0$ be given. Then for every $0 \leq m \leq N-1$ there exists a constant $C_{m}>0$, depending only on $N$ and $\delta$, such that for all $\Lambda=\left(\lambda_{1}, \ldots, \lambda_{N+1}\right)$ with the property that $\left|e^{\lambda_{j}}-1\right| \geq \delta$ for all $j=1, \ldots, N+1$, the following inequality

$$
\left|\frac{d^{m}}{d x^{m}} Q_{\Lambda}(x)\right| \leq C_{m} e^{-\left(\lambda_{1}+\ldots+\lambda_{N+1}\right)} M_{\Lambda}^{m} \cdot B_{\Lambda}(m) \cdot\left|r_{\Lambda}(1)\right|
$$

holds for all $x \in \mathbb{R}$.

PROOF. Let us prove the claim at first for the case $m=0$ : The basis spline $Q_{\Lambda}$ is non-negative and it has support in $[0, N+1]$; for $y \in[0, N+1]$ we can find $j \in\{0,1, \ldots, M\}$ and $x \in[0,1]$ with $y=x+j$. Clearly

$$
Q_{\Lambda}(y) \leq \sum_{j=0}^{N} Q_{\Lambda}(x+j)
$$

Taking $\lambda=1$ in formula (11), one obtains that

$$
Q_{\Lambda}(y) \leq \frac{\left|\Pi_{\Lambda}(x, 1)\right|}{e^{\left(\lambda_{1}+\ldots+\lambda_{N+1}\right)}}=\frac{1}{e^{\left(\lambda_{1}+\ldots+\lambda_{N+1}\right)}}\left|A_{\Lambda}(x, 1) \cdot r_{\Lambda}(1)\right|
$$

Hence the claim is true for $m=0$ where $C_{0}=1$.

We proceed by induction over $m=0, \ldots, N-1$ and assume that the statement is true for $m \leq N-1$. If $m=N-1$ we are done, so assume that $m<N-1$. We apply the induction hypothesis to $\Lambda=\left(\lambda_{1}, \ldots, \lambda_{N+1}\right)$ and $\Lambda_{2}=\left(\lambda_{1}, \ldots, \lambda_{N}\right)$ (note that $m \leq N-2$ ), hence for all $x \in \mathbb{R}$

$$
\begin{aligned}
\left|\frac{d^{m}}{d x^{m}} Q_{\Lambda}(x)\right| & \leq C_{1} e^{-\left(\lambda_{1}+\ldots+\lambda_{N+1}\right)} M_{\Lambda}^{m} \cdot B_{\Lambda}(m) \cdot\left|r_{\Lambda}(1)\right| \\
\left|\frac{d^{m}}{d x^{m}} Q_{\Lambda_{2}}(x)\right| & \leq C_{2} e^{-\left(\lambda_{1}+\ldots+\lambda_{N}\right)} M_{\Lambda_{2}}^{m} \cdot B_{\Lambda_{2}}(m) \cdot\left|r_{\Lambda_{2}}(1)\right|
\end{aligned}
$$


In $[7$, p. 119] or $[10$, Part II] one can find the formula

$$
\begin{gathered}
\frac{d}{d x} Q_{\left(\lambda_{1}, \ldots, \lambda_{N+1}\right)}(x)=\lambda_{N+1} Q_{\left(\lambda_{1}, \ldots, \lambda_{N+1}\right)}(x)+e^{-\lambda_{N+1}} Q_{\left(\lambda_{1}, \ldots, \lambda_{N}\right)}(x) \\
+Q_{\left(\lambda_{1}, \ldots, \lambda_{N}\right)}(x-1) .
\end{gathered}
$$

Differentiating the last equation $m$ times yields

$$
\begin{gathered}
\frac{d^{m+1}}{d x^{m+1}} Q_{\Lambda}(x)=\lambda_{N+1} \frac{d^{m}}{d x^{m}} Q_{\left(\lambda_{1}, \ldots, \lambda_{N+1}\right)}(x)+e^{-\lambda_{N+1}} \frac{d^{m}}{d x^{m}} Q_{\left(\lambda_{1}, \ldots, \lambda_{N}\right)}(x) \\
+\frac{d^{m}}{d x^{m}} Q_{\left(\lambda_{1}, \ldots, \lambda_{N}\right)}(x-1)
\end{gathered}
$$

The triangle inequality and our induction hypothesis show that

$$
\begin{aligned}
\left|\frac{d^{m+1}}{d x^{m+1}} Q_{\Lambda}(x)\right| \leq & \left|\lambda_{N+1}\right| C_{1} e^{-\left(\lambda_{1}+\ldots+\lambda_{N+1}\right)} M_{\Lambda}^{m} \cdot B_{\Lambda}(m) \cdot\left|r_{\Lambda}(1)\right|+ \\
& \left(e^{-\lambda_{N+1}}+1\right) C_{2} e^{-\left(\lambda_{1}+\ldots+\lambda_{N}\right)} M_{\Lambda_{2}}^{m} \cdot B_{\Lambda_{2}}(m) \cdot\left|r_{\Lambda_{2}}(1)\right| .
\end{aligned}
$$

Now $r_{\left(\lambda_{1}, \ldots, \lambda_{N+1}\right)}(1)=\left(e^{\lambda_{N+1}}-1\right) r_{\left(\lambda_{1}, \ldots, \lambda_{N}\right)}(1)$ and $\left|\lambda_{N+1}\right| \leq M_{\Lambda}$, and $M_{\Lambda_{2}}^{m} \leq$ $M_{\Lambda}^{m}$. Thus

$$
\left|\frac{d^{m+1}}{d x^{m+1}} Q_{\Lambda}(x)\right| \leq e^{-\left(\lambda_{1}+\ldots+\lambda_{N+1}\right)}\left|r_{\Lambda}(1)\right| \cdot M_{\Lambda}^{m+1} \cdot C_{\Lambda}
$$

where

$$
C_{\Lambda}=\left(C_{1} B_{\Lambda}(m)+C_{2} \frac{1}{M_{\Lambda}} B_{\Lambda_{2}}(m) \frac{\left(e^{-\lambda_{N+1}}+1\right) e^{\lambda_{N+1}}}{\left|e^{\lambda_{N+1}}-1\right|}\right)
$$

Further we have the trivial estimate $B_{\Lambda}(m) \leq B_{\Lambda}(m+1)$ and

$$
B_{\Lambda_{2}}(m)=\sum_{k=1}^{m+1} \max _{0 \leq x \leq 1}\left|M_{\Lambda}^{-(k-1)} A_{\left(\lambda_{1}, \ldots, \lambda_{N+1-k}\right)}(x, 1)\right| \leq M_{\Lambda} B_{\Lambda}(m+1)
$$

The function $x \longmapsto\left|(x+1)(x-1)^{-1}\right|$ is bounded on $\mathbb{R} \backslash[1-\delta, 1+\delta]$. Since $\left|e^{\lambda_{j}}-1\right| \geq \delta$ for all $j=1, \ldots, N+1$, we infer $C_{\Lambda} \leq C_{3} B_{\Lambda}(m+1)$ where $C_{3}$ depends only on $N$ and $\delta$. The proof is complete. 


\subsection{Symmetry properties}

Let $\Lambda=\left(\lambda_{1}, \ldots, \lambda_{N+1}\right)$ and define $-\Lambda=\left(-\lambda_{1}, \ldots,-\lambda_{N+1}\right)$. For all $x \in \mathbb{R}$ and $\lambda \notin e^{\Lambda} \cup e^{-\Lambda} \cup\{0\}$ the following identity (see [17, p. 213])

$$
A_{\Lambda}\left(1-x, \frac{1}{\lambda}\right)=(-1)^{N+1} \lambda \cdot A_{-\Lambda}(x, \lambda)
$$

follows by a direct computation. As in [11] we call $\Lambda$ nearly symmetric if there exists $c \in \mathbb{R}$ and a permutation $\pi$ of the set $\{1, \ldots, N+1\}$ such that $-\lambda_{j}=c+\lambda_{\pi(j)}$ for $j=1, \ldots, N+1$, or shortly $-\Lambda=c+\Lambda$. In the case $c=0$ we call $\Lambda$ symmetric. Note that for $j \in\{1, \ldots, N+1\}$ with $\pi(j)=j$ one obtains that $-c=\lambda_{j}+\lambda_{\pi(j)}=2 \lambda_{j}$ and therefore $\lambda_{j}=-\frac{1}{2} c$. It follows that

$$
\lambda_{1}+\ldots+\lambda_{N+1}=-\frac{1}{2}(N+1) c
$$

since $\lambda_{j}+\lambda_{\pi(j)}=-c$ for $j=1, \ldots N+1$. A simple computation shows that for all $x \in \mathbb{R}$ and $\lambda \notin e^{\Lambda} \cup e^{-\Lambda} \cup\{0\}$

$$
A_{-\Lambda}(x, \lambda)=e^{(x-1) c} A_{\Lambda}\left(x, \lambda e^{-c}\right)
$$

Combining equation (20) and (22) one obtains

Proposition 7 Let $\Lambda$ be nearly symmetric with respect to $c \in \mathbb{R}$. For all $\lambda \notin e^{\Lambda} \cup e^{-\Lambda} \cup\{0\}$ and all $x \in \mathbb{R}$ the following equality

$$
A_{\Lambda}\left(1-x, \frac{1}{\lambda}\right)=(-1)^{N+1} \lambda e^{(x-1) c} A_{\Lambda}\left(x, \lambda e^{-c}\right)
$$

holds.

Similiar computations lead to the following result (cf. Proposition 7 in [11]):

Proposition 8 Let $\Lambda$ be nearly symmetric with respect to $c \in \mathbb{R}$. Then the polynomial $P_{\Lambda}(\lambda)$ defined in (13) is given by

$$
P_{\Lambda}(\lambda)=(-1)^{N} \lambda e^{N c} \cdot \Pi_{\Lambda}\left(0, \lambda e^{-c}\right)
$$




\section{Estimate of the function $A_{\Lambda}(x, \lambda)$}

In this Section we will give an estimate of the asymptotic behavior of the function $A_{\Lambda(k)}(x, \lambda)$ for $k \rightarrow \infty$ and $0 \leq x \leq 1$. This estimate will be used to prove the existence of an interpolation polyspline for the case that $\Lambda=\Lambda(k)$ is of the form (4).

Assume that for each $k \in \mathbb{N}_{0}$ the vector $\Lambda=\Lambda(k)=\left\{\lambda_{1}(k), \ldots, \lambda_{N+1}(k)\right\}$ is of the following form: there exists $r \in\{1, \ldots, N+1\}$ (independent of $k \in$ $\mathbb{N}_{0}$ ), pairwise different real numbers $C_{1}, . ., C_{r}$, and pairwise different numbers $C_{r+1}, \ldots, C_{N+1}$, such that for all $k \in \mathbb{N}_{0}$ we have the equalities

$$
\lambda_{j}=\lambda_{j}(k)=\left\{\begin{array}{l}
-k+C_{j} \text { for } j=1, \ldots, r \\
k+C_{j} \quad \text { for } j=r+1, \ldots, N+1 .
\end{array}\right.
$$

Then for large $k$ all $\lambda_{j}(k)$ are pairwise different for $j=1, \ldots, N+1$, consequently

$$
A_{\Lambda(k)}(x, \lambda)=\sum_{j=1}^{N+1} \frac{1}{q_{\Lambda(k)}^{\prime}\left(\lambda_{j}(k)\right)} \frac{e^{\lambda_{j}(k) x}}{e^{\lambda_{j}(k)}-\lambda}
$$

where $q_{\Lambda(k)}^{\prime}$ is the derivative of $q_{\Lambda(k)}$. Let us split $A_{\Lambda(k)}(x, \lambda)$ into a sum of two functions

$$
\begin{aligned}
& c_{k}(x, \lambda)=\sum_{j=1}^{r} \frac{1}{q_{\Lambda}^{\prime}\left(\lambda_{j}(k)\right)} \frac{e^{\lambda_{j}(k) x}}{e^{\lambda_{j}}-\lambda} \\
& d_{k}(x, \lambda)=\sum_{j=r+1}^{N+1} \frac{1}{q_{\Lambda}^{\prime}\left(\lambda_{j}(k)\right)} \frac{e^{\lambda_{j}(x) x}}{e^{\lambda_{j}}-\lambda} .
\end{aligned}
$$

Let $K$ be a compact subset of the complex plane such that $0 \notin K$ and let $\delta$ be a positive number. Then it is easy to see that the sequence $\left(d_{k}(x, \lambda)\right)_{k \in \mathbb{N}_{0}}$ with $\lambda \in K$ and $0 \leq x \leq 1-\delta$ is of uniform exponential decay in the following sense: there exists a polynomial $P$ and $\varepsilon>0$ such that $\left|d_{k}(x, \lambda)\right| \leq|P(k)| \cdot e^{-\varepsilon \cdot k}$ for all $k \in \mathbb{N}_{0}$, all $\lambda \in K$, and all $0 \leq x \leq 1-\delta$.

Let us define

$$
b_{k}(x)=\sum_{j=1}^{r} \frac{e^{\lambda_{j}(k) x}}{q_{\Lambda}^{\prime}\left(\lambda_{j}(k)\right)} .
$$


The following simple result tells us that the asymptotic of $\lambda A_{\Lambda(k)}(x, \lambda)$ for $k \rightarrow \infty$ is the same as of $b_{k}(x)$.

Proposition 9 Define $E(k, \lambda):=\prod_{l=1}^{r}\left(e^{\lambda_{l}(k)}-\lambda\right)$ and let $K$ be a compact subset of the complex plane not containing 0 and let $0<\delta<1$. Then we can write

$$
\lambda A_{\Lambda}(x, \lambda)=\frac{(-\lambda)^{r}}{E(k, \lambda)} b_{k}(x)+\lambda f_{k}(x, \lambda)
$$

where $f_{k}(x, \lambda)$ is of uniform exponential decay on $[0,1-\delta]$ and $E(k, \lambda)$ converges uniformly on $K$ to $(-\lambda)^{r} \neq 0$.

PROOF. Define $E_{j}(k, \lambda):=\prod_{l=1, l \neq j}^{r}\left(e^{\lambda_{l}(k)}-\lambda\right)$. Then $E_{j}(k, \lambda)$ is a sum of sequences of uniform exponential decay and the constant $(-\lambda)^{r-1}$. It is easy to see that

$$
e_{k}(x, \lambda):=\left(\sum_{j=1}^{r} \frac{e^{\lambda_{j}(k) x}}{q_{\Lambda}^{\prime}\left(\lambda_{j}(k)\right)} E_{j}(k, \lambda)\right)-(-\lambda)^{r-1} b_{k}(x)
$$

is of uniform exponential decay. Thus

$$
f_{k}(x, \lambda):=\frac{e_{k}(x, \lambda)}{E(k, \lambda)}+d_{k}(x, \lambda)=A_{\Lambda}(x, \lambda)-\frac{(-\lambda)^{r-1}}{E(k, l)} b_{k}(x)
$$

is of uniform exponential decay.

Theorem 10 Let $\Lambda(k)$ be as in (25) and let $K$ be a compact subset of the complex plane with $0 \notin K$. Then for each $\delta>0$ there exists a constant $D>0$ and a natural number $k_{0}$ such that for all $k \geq k_{0}$, all $\lambda \in K$, and all $0 \leq x \leq$ $1-\delta$ the following estimate

$$
\left|A_{\Lambda(k)}(x, \lambda)\right| \leq D \frac{1}{k^{N}}
$$

holds. If there exists $c \in \mathbb{R}$ such that $\Lambda(k)$ is nearly symmetric with respect to $c$ for all $k \geq k_{0}$ then the inequality is valid for all $0 \leq x \leq 1$.

PROOF. We may assume that $K$ is disjoint with $e^{\Lambda(k)}$ for large $k$. Let $\gamma(t)=$ $e^{i t}$ for $t \in[0,2 \pi]$ and define $\Gamma_{k}(t):=-k+k \gamma(t)$. Let $k_{0} \in \mathbb{N}_{0}$ be so large that 
$\left|C_{j}\right|<\frac{1}{2} k_{0}$ for all $j=1, \ldots, N+1$. Then for all $k \geq k_{0}$ the curve $\Gamma_{k}$ surrounds $\lambda_{1}, \ldots, \lambda_{r}$ but not $\lambda_{r+1}, \ldots, \lambda_{N+1}$. By Cauchy's Theorem

$$
b_{k}(x)=\sum_{j=1}^{r} \frac{e^{\lambda_{j} x}}{q_{\Lambda}^{\prime}\left(\lambda_{j}\right)}=\frac{1}{2 \pi i} \int_{\Gamma_{k}} \frac{e^{z x}}{q_{\Lambda}(z)} d z .
$$

Note that $\left|\lambda_{j}-z\right| \geq k-\frac{1}{2} k_{0} \geq \frac{1}{2} k$ for all $z$ on the path $\Gamma_{k}$ and for all $j=1, \ldots, N+1$. Clearly $\left|e^{z x}\right| \leq e^{x \operatorname{Re}(z)}$ (assuming $0 \leq x \leq 1$ ) is bounded for $z \in \Gamma_{k}$. Hence the standard estimate for line integrals gives for a suitable constant $M>0$ the inequality

$$
\left|b_{k}(x)\right| \leq M \frac{1}{k^{N+1}} k
$$

for all $0 \leq x \leq 1$ and $k \geq k_{0}$. By (28) we have uniform exponential decay for $\left(\lambda f_{k}(x, \lambda)\right)_{k \in \mathbb{N}_{0}}$, i.e. there exists a polynomial $P$ and $\varepsilon>0$ such that $\left|\lambda f_{k}(x, \lambda)\right| \leq|P(k)| \cdot e^{-\varepsilon \cdot k}$ for all $k \in \mathbb{N}_{0}$, all $0 \leq x \leq 1-\delta$, and all $\lambda \in K$. Since $\frac{(-\lambda)^{r}}{E(k, \lambda)}$ converges uniformly to 1 it follows that for large $k$

$$
\left|\lambda A_{\Lambda}(x, \lambda)\right| \leq\left|\frac{(-\lambda)^{r}}{E(k, \lambda)} b_{k}(x)\right|+\left|\lambda f_{k}(x, \lambda)\right| \leq 2 M \frac{1}{k^{N}}+|P(k)| \cdot e^{-\varepsilon \cdot k}
$$

and (29) is proven for $0 \leq x \leq 1-\delta$.

For the second statement let $K_{1}:=K \cup\left\{1 / \lambda e^{c}: \lambda \in K\right\}$ and let $\delta=1 / 4$. Then there exists a constant $D>0$ such that $\left|A_{\Lambda(k)}(x, \mu)\right| \leq D \frac{1}{k^{N}}$ for all $0 \leq x \leq 1-\delta$ and for all $\mu \in K_{1}$. Let now $\frac{1}{2} \leq y \leq 1$ and define $x=1-y$. By equation (23), (replace $\lambda$ by $\lambda e^{c}$ and $x$ by $y$ and note that $N+1=2 p$ )

$$
A_{\Lambda}(y, \lambda)=\frac{1}{\lambda e^{c}} e^{-(y-1) c} A_{\Lambda}\left(1-y, \frac{1}{\lambda e^{c}}\right)=\frac{1}{\lambda e^{c}} e^{x c} A_{\Lambda}\left(x, \frac{1}{\lambda e^{c}}\right) .
$$

Hence $\left|A_{\Lambda}(y, \lambda)\right| \leq D_{2} D \frac{1}{k^{N}}$ for all $\frac{1}{2} \leq y \leq 1$ and the proof is complete.

Theorem 11 Let $\Lambda(k)$ be as in (25) and let $K$ be a compact subset of the complex plane with $0 \notin K$. If $r<N+1$ then there exist constants $C, D>0$ and a natural number $k_{0}$ such that for all $k \geq k_{0}$ and all $\lambda \in K$

$$
C \frac{1}{k^{N}} \leq\left|A_{\Lambda(k)}(0, \lambda)\right| \leq D \frac{1}{k^{N}} .
$$

Further the following inequality holds for all $\lambda \in(-\infty, 0) \cap K$ and all $k \geq k_{0}$,

$$
(-1)^{N+r} A_{\Lambda(k)}(0, \lambda)>0 .
$$


PROOF. Note that by (30)

$$
k^{N} b_{k}(x)=\frac{1}{2 \pi i} \int_{0}^{2 \pi} \frac{e^{-k x(1-\gamma(t))} \cdot \gamma^{\prime}(t)}{\prod_{j=1}^{r}\left(\gamma(t)-\frac{C_{j}}{k}\right) \prod_{j=r+1}^{N+1}\left(-2+\gamma(t)-\frac{C_{j}}{k}\right)} d t .
$$

Clearly the denominator of the integrand converges to $(\gamma(t))^{r}(\gamma(t)-2)^{N+1-r}$. For $x=0$ the nominator is trivially convergent and hence we see that $k^{N} b_{k}(0)$ converges to

$$
d_{r}:=\frac{1}{2 \pi i} \int_{\gamma} \frac{1}{z^{r}(z-2)^{N+1-r}} d z .
$$

Since $\gamma$ surrounds $z=0$ but not $z=2$ this value can be computed by residue theory (see e.g. Proposition 2.4 in [6, p. 113]) and we obtain

$$
d_{r}=\frac{(-1)^{r-1+N}}{(r-1) !} 2^{N}(N+1-r) \ldots(N-1) .
$$

It follows that there exist a constant $C>0$ and an integer $k_{0}$ such that $(-1)^{r-1+N} b_{k}(0) \geq C \frac{1}{k^{N}}$ for all $k \geq k_{0}$.

Assume now that $K \subset(-\infty, 0)$. Since for $k \longrightarrow \infty$ we have $\frac{(-\lambda)^{r}}{E(k, \lambda)} \longrightarrow 1$ uniformly on $K$, there exists an integer $k_{1}$ such that for all $k \geq k_{1}$ and all $\lambda \in K$

$$
\frac{(-\lambda)^{r}}{E(k, \lambda)}(-1)^{r-1+N} b_{k}(0) \geq \frac{C}{2} \frac{1}{k^{N}}>0 .
$$

Since the sequence $\left(\lambda f_{k}(0, \lambda)\right)_{k \in \mathbb{N}_{0}}$ is of uniform exponential decay there exists a polynomial $P$ and a number $\varepsilon>0$ such that $\left|\lambda f_{k}(0, \lambda)\right| \leq|P(k)| \cdot e^{-\varepsilon \cdot k}$ for all $k \in \mathbb{N}_{0}$ and for all $\lambda \in K$. Then by (27) the following inequalities hold:

$$
\begin{gathered}
(-1)^{r-1+N} \lambda A_{\Lambda}(0, \lambda) \geq \frac{(-\lambda)^{r}}{E(k, \lambda)}(-1)^{r-1+N} b_{k}(0)-\left|\lambda f_{k}(0, \lambda)\right| \\
\geq \frac{C}{2} \frac{1}{k^{N}}-|P(k)| \cdot e^{-\varepsilon \cdot k} \geq \frac{1}{4} \frac{C}{k^{N}}
\end{gathered}
$$

for all sufficiently large $k$ and for all $\lambda \in K$. Since the set $K$ contains only negative numbers we obtain the estimate (32) for all sufficiently large $k$.

Now assume that $K$ is a compact subset in the complex plane $\mathbb{C}$. Then similar arguments as above show that for some $k_{1} \in \mathbb{N}_{0}$ the inequality $\left|\lambda A_{\Lambda}(0, \lambda)\right| \geq$ $\frac{1}{4} \frac{C}{k^{N}}$ holds for all $\lambda \in K$, and for all $k \geq k_{1}$. 


\section{Uniform estimates of fundamental $L$-splines}

In the rest of the paper we will assume that $\Lambda(k)$ is given by (4). We write $\lambda_{j}(k)=-k+C_{j}$ for $j=1, \ldots, p$ with

$$
C_{1}=2-n, C_{2}=4-n, \ldots, C_{p}=2 p-n,
$$

and $\lambda_{j}(k)=k+C_{j}$ for $j=p+1, \ldots, 2 p$ with

$$
C_{p+1}=0, C_{p+2}=2, \ldots, C_{2 p}=2 p-2 .
$$

Hence $N+1=2 p$ and clearly $\Lambda(k)$ is nearly symmetric with respect to $c=n-2 p$ where $n \in \mathbb{N}_{0}$ is the dimension of the underlying space $\mathbb{R}^{n}$.

Theorem 12 Let $\Lambda(k)$ be as in (4) and let $K$ be a compact subset of the complex plane with $0 \notin K$. Then there exist a constant $M>0$ and an integer $k_{0}$ such that $P_{\Lambda(k)}(\lambda) \neq 0$ for all $k \geq k_{0}$ and for all $\lambda \in K$; further for all $k \geq k_{0}$

$$
C(k):=\max _{x \in[0,1]} Q_{\Lambda(k)}(x) \cdot \max _{\lambda \in K} \frac{1}{\left|P_{\Lambda(k)}(\lambda)\right|} \leq M .
$$

More generally, for every $m=0, \ldots, 2 p-2$ there exist a constant $M_{1}>0$ and an integer $k_{1}$ such that for all $\lambda \in K$ and for all $k \geq k_{1}$

$$
C_{m}(k):=\max _{x \in[0,1]}\left|\frac{d^{m}}{d x^{m}} Q_{\Lambda(k)}(x)\right| \cdot \max _{\lambda \in K} \frac{1}{\left|P_{\Lambda(k)}(\lambda)\right|} \leq M_{1} k^{m} .
$$

PROOF. Using $N+1=2 p$ and $c=n-2 p$ Proposition 8 yields

$$
P_{\Lambda(k)}(\lambda)=(-1) \lambda e^{N c} A_{\Lambda(k)}\left(0, \lambda e^{-c}\right) \cdot r_{\Lambda(k)}\left(\lambda e^{-c}\right)
$$

where $r_{\Lambda(k)}(\lambda)=\prod_{j=1}^{2 p}\left(e^{\lambda_{j}(k)}-\lambda\right)$. By Theorem 11 applied to the compact set $e^{-c} K:=\left\{e^{-c} \lambda: \lambda \in K\right\}$ there exists $C>0$ and $k_{0} \in \mathbb{N}_{0}$ such that $C \leq$ $\left|A_{\Lambda(k)}\left(0, \lambda e^{-c}\right)\right| \cdot k^{2 p-1}$ for all $\lambda \in K$ and for all $k \geq k_{0}$. Thus by $(36) P_{\Lambda(k)}(\lambda) \neq$ 0 for all $\lambda \in K$ and for all $k \geq k_{0}$ and the first statement is proven. Furthermore we have obtained the estimate

$$
\frac{1}{\left|P_{\Lambda(k)}(\lambda)\right|} \leq \frac{e^{-N c}}{C|\lambda|} k^{2 p-1} \frac{1}{r_{\Lambda(k)}\left(\lambda e^{-c}\right)}
$$


In order to prove (34) we apply Theorem 6 with $m=0$, and obtain

$$
\left|Q_{\Lambda(k)}(x)\right| \leq C e^{-\left(\lambda_{1}+\ldots+\lambda_{N+1}\right)} \max _{0 \leq y \leq 1}\left|A_{\Lambda(k)}(y, 1)\right| \cdot\left|r_{\Lambda(k)}(1)\right|
$$

Theorem 10 shows that there exists $D_{1}>0$ such that

$$
\max _{x \in[0,1]} Q_{\Lambda(k)}(x) \leq D_{1} e^{p(n-2 p)} \frac{1}{k^{2 p-1}}\left|r_{\Lambda(k)}(1)\right| .
$$

Hence we obtain for a suitable constant $D_{2}$ (note that $\left.0 \notin K\right)$ the inequality

$$
C(k) \leq D_{2}\left|r_{\Lambda(k)}(1)\right| \max _{\lambda \in K} \frac{1}{\left|r_{\Lambda(k)}\left(\lambda e^{-c}\right)\right|}
$$

The proof is accomplished by the fact that

$$
\frac{r_{\Lambda(k)}(1)}{r_{\Lambda(k)}\left(\lambda e^{-c}\right)}=\frac{\prod_{k=1}^{p}\left(e^{-k+C_{j}}-1\right) \prod_{k=p+1}^{2 p}\left(e^{k+C_{j}}-1\right)}{\prod_{k=1}^{p}\left(e^{-k+C_{j}}-\lambda e^{-c}\right) \prod_{k=p+1}^{2 p}\left(e^{k+C_{j}}-\lambda e^{-c}\right)}
$$

converges uniformly for $k \rightarrow \infty$ to $\frac{1}{\left(\lambda e^{-c}\right)^{p}}$. The estimate (35) follows in the same way using again Theorem 6 and Theorem 10.

For the proof of our main result we need the following Proposition which establishes an uniform estimate of the type (12) of all fundamental splines for the operators $L$ generated by the vectors $\Lambda(k)$.

Proposition 13 For every $k \in \mathbb{N}_{0}$ let $\Lambda(k)$ be as in (4). Then there exists a fundamental $L-$ spline $L_{\Lambda(k)}$ with respect to the operator $M_{\Lambda(k)}$. Further there exist constants $M>0$ and $\varepsilon>0$ such that for all $k \in \mathbb{N}_{0}$ and all $v \in \mathbb{R}$ the following estimate holds:

$$
\left|L_{\Lambda(k)}(v)\right| \leq M e^{-\varepsilon|v|}
$$

PROOF. At first we show that $A_{\Lambda(k)}(0,-1) \neq 0$ for all $k \in \mathbb{N}_{0}$. The integral

$$
A_{\Lambda(k)}(0,-1)=\frac{1}{2 \pi i} \int_{\Gamma} \frac{1}{q_{\Lambda(k)}(z)} \frac{1}{e^{z}+1} d z
$$

can be computed by residue theory and it reduces to a rational expression which has a non-zero denominator. For simplicity let us consider the case 
when the constants $\lambda_{j}(k)$ are pairwise distinct. Then

$$
A_{\Lambda(k)}(0,-1)=\sum_{j=1}^{2 p} \frac{1}{q_{\Lambda(k)}^{\prime}\left(\lambda_{j}(k)\right)} \frac{1}{e^{\lambda_{j}(k)}+1} .
$$

Obviously, $q_{\Lambda(k)}^{\prime}\left(\lambda_{j}(k)\right)$ are integers. Let us assume that $A_{\Lambda(k)}(0,-1)=0$. After multiplying by $\prod_{j=1}^{2 p}\left(e^{\lambda_{j}(k)}+1\right)$ we arrive at an equation of the type

$$
\sum_{i=1}^{l} \beta_{i} e^{\rho_{i}}=0
$$

here $\beta_{i}$ are non-zero rationals and $\rho_{i}$ are integers obtained by sums of some of the constants $\lambda_{j}(k)$. Due to the special form of the constants $\lambda_{j}(k)$ provided in (4) at least one of the $\rho_{i}$ is non-zero. Thus we may apply the classical theorem of Lindemann on transcendental numbers which states that the above equality is impossible, see e.g. K. Mahler [15, p. 213] or A. Baker [3, p. 6]. It follows that $A_{\Lambda(k)}(0,-1) \neq 0$.

By Theorem 3 we can find for each $k \in \mathbb{N}_{0}$ a fundamental $L$-spline $L_{\Lambda(k)}: \mathbb{R} \rightarrow$ $\mathbb{R}$. Hence, there exist constants $M_{k}$ and $\varepsilon_{k}$ such that for all $v \in \mathbb{R}$ holds

$$
\left|L_{\Lambda(k)}(v)\right| \leq M_{k} e^{-\varepsilon_{k}|v|}
$$

We have to show that the constants $M_{k}$ can be chosen as a bounded sequence, and similarly that $\varepsilon_{k} \geq \varepsilon$ for all $k \in \mathbb{N}_{0}$. Let $0<\rho<1$ and put $K:=$ $\{\lambda \in \mathbb{C}: \rho \leq|\lambda| \leq 1 / \rho\}$. Choose arbitrary $\rho^{*}$ with $0<\rho^{*}<\rho$ and put $T:=$ $\left\{\lambda \in \mathbb{C}: \rho^{*} \leq|\lambda| \leq 1 / \rho^{*}\right\}$. By Theorem 12 applied to the compact set $T$ there exists $k_{0} \in \mathbb{N}_{0}$ such that

$$
P_{\Lambda(k)}(\lambda) \neq 0
$$

for all $\lambda \in T$ and for all $k \geq k_{0}$. Hence $P_{\Lambda(k)}$ is holomorphic on the open annulus given by the radii $R_{1}=\rho^{*}<1<1 / \rho^{*}=R_{2}$ for all $k \geq k_{0}$. Again by Theorem 12 applied to the compact set $K$ there exist a constant $M^{*}>0$ and a natural number $k_{1} \geq k_{0}$ such that

$$
C(k):=\max _{x \in[0,1]} Q_{\Lambda(k)}(x) \cdot \max _{\lambda \in K} \frac{1}{\left|P_{\Lambda(k)}(\lambda)\right|} \leq M^{*}
$$

for all $k \geq k_{1}$. Apply now Proposition 5 with respect to all sets $\Lambda(k)$ with $k \geq k_{1}$. It follows that there exists a constant $G(\rho)$ (independent of $k$ ) such 
that the fundamental $L$-splines $L_{\Lambda(k)}$ for $k \geq k_{1}$ can be estimated by

$$
\left|L_{\Lambda(k)}(v)\right| \leq G(\rho) C(k) e^{-\varepsilon^{*}|v|} \leq G(\rho) M^{*} e^{-\varepsilon^{*}|v|}
$$

where $\varepsilon^{*}:=-\log \rho$. Finally after putting $M:=\max \left\{M^{*}, M_{0}, \ldots, M_{k_{1}-1}\right\}$ and $\varepsilon:=\min \left\{\varepsilon^{*}, \varepsilon_{0}, \ldots, \varepsilon_{k_{1}-1}\right\}$ the proof is complete.

\section{The main result}

At first we need some notations: assume that the function $f: \mathbb{S}^{n-1} \rightarrow \mathbb{R}$ be square-integrable with respect to the surface measure $d \theta$ on $\mathbb{S}^{n-1}$ and define the usual scalar product

$$
\langle f, g\rangle_{L^{2}\left(\mathbb{S}^{n-1}\right)}=\int_{\mathbb{S}^{n-1}} f(\theta) \overline{g(\theta)} d \theta .
$$

Recall that $Y_{k, l}(\theta)$, for $k \in \mathbb{N}_{0}, l=1, \ldots, a_{k}$ denotes an orthonormal basis of the space $\mathcal{H}_{k}$ of all spherical harmonics with respect to $d \theta$. For all $k \in \mathbb{N}_{0}$, and $l=1, \ldots, a_{k}$ the Fourier-Laplace coefficients of $f$ are given by

$$
f_{k, l}:=\int_{\mathbb{S}^{n-1}} f(\theta) Y_{k, l}(\theta) d \theta .
$$

By [23, Corollary 2.3] every square-integrable function $f$ can be expanded into a Fourier-Laplace series given by

$$
f(\theta)=\sum_{k=0}^{\infty} \sum_{l=1}^{a_{k}} f_{k, l} \cdot Y_{k, l}(\theta)
$$

where convergence is understood in $L_{2}\left(\mathbb{S}^{n-1}\right)$ with the norm

$$
\|f\|_{L_{2}\left(\mathbb{S}^{n-1}\right)}=\sqrt{\langle f, f\rangle_{L_{2}\left(\mathbb{S}^{n-1}\right)}} .
$$

For every $f \in L_{2}\left(\mathbb{S}^{n-1}\right)$ define

$$
\|f\|_{s}:=\sum_{k=0}^{\infty} \sum_{l=1}^{a_{k}}\left|f_{k, l}\right| \cdot(1+k)^{s} .
$$

The subspace of all $f \in L_{2}\left(\mathbb{S}^{n-1}\right)$ with $\|f\|_{s}<\infty$ is denoted by $H^{s, 1}\left(\mathbb{S}^{n-1}\right)$, see [1]. 
By [21], for all $Y_{k} \in \mathcal{H}_{k}$ we have the inequality

$$
\left|Y_{k}(\theta)\right| \leq K k^{(n / 2)-1}\left\|Y_{k}(\theta)\right\|_{L_{2}\left(\mathbb{S}^{n-1}\right)} \quad \text { for } \theta \in \mathbb{S}^{n-1}
$$

Since $\left\|Y_{k, l}(\theta)\right\|_{L_{2}\left(\mathbb{S}^{n-1}\right)}=1$ we obtain the estimate

$$
\sum_{k=0}^{\infty} \sum_{l=1}^{a_{k}}\left|f_{k, l}\right|\left|Y_{k, l}(\theta)\right| \leq K \sum_{k=0}^{\infty} \sum_{l=1}^{a_{k}}\left|f_{k, l}\right|(1+k)^{\frac{n}{2}-1}=K\|f\|_{\frac{n}{2}-1} .
$$

It follows that a function $f \in H^{\frac{n}{2}-1}\left(\mathbb{S}^{n-1}\right)$ possesses an absolutely uniformly convergent Fourier-Laplace series.

Using some standard techniques (see e.g. [8]) one can prove the following criterion:

Proposition 14 Assume that $f: \mathbb{S}^{n-1} \rightarrow \mathbb{R}$ is a $2 q$-continuously differentiable function where $2 q \geq 2(p-1)+2\left[\frac{n}{2}\right]$. Then $f \in H^{s, 1}\left(\mathbb{S}^{n-1}\right)$ for $s=2(p-1)+$ $(n / 2)-1$.

\subsection{Construction of fundamental polysplines}

As in the one-dimensional case we show at first the existence of "fundamental polysplines" in the following sense:

Definition $15 A$ fundamental polyspline $L_{f}: \mathbb{R}^{n} \backslash\{0\} \rightarrow \mathbb{R}$ for the data function $f: \mathbb{S}^{n-1} \rightarrow \mathbb{C}$ is the polyspline of order $p$ such that for each $j \in \mathbb{Z}$ the interpolation conditions

$$
\begin{aligned}
& L_{f}\left(e^{j} \theta\right)=0 \quad \text { for all } j \neq 0 \text { and } \theta \in \mathbb{S}^{n-1}, \\
& L_{f}\left(e^{j} \theta\right)=f(\theta) \text { for } j=0 \text { and all } \theta \in \mathbb{S}^{n-1}
\end{aligned}
$$

hold, as well as the following growth condition

$$
\left|L_{f}(r \theta)\right| \leq M e^{-\varepsilon|\log r|} \quad \text { for all } r>0 \text { and } \theta \in \mathbb{S}^{n-1} .
$$

The next result ensures the existence of fundamental polysplines for a large class of data functions.

Theorem 16 Let $s=s_{p, n}=2(p-1)+(n / 2)-1$. Then there exist constants $M>0$ and $\varepsilon>0$ with the following property: for each $f \in H^{s, 1}\left(\mathbb{S}^{n-1}\right)$ there 
exists a polyspline $L_{f}$ of order $p$ such that (43) holds and

$$
\left|\frac{d^{m}}{d r^{m}} D^{\alpha} L_{f}(r \theta)\right| \leq M e^{-\varepsilon|\log r|}\|f\|_{s}
$$

for all $m \in \mathbb{N}_{0}$ and $\alpha=\left(\alpha_{1}, \ldots, \alpha_{n-1}\right) \in \mathbb{N}_{0}^{n-1}$ satisfing the condition $m+|\alpha| \leq$ $2 p-2$; here $D^{\alpha}$ denotes the differential operator

$$
D^{\alpha}:=\frac{\partial^{\alpha_{1}}}{\partial \theta_{1}^{\alpha_{1}}} \cdots \frac{\partial^{\alpha_{n-1}}}{\partial \theta_{n-1}^{\alpha_{n-1}}}
$$

PROOF. Let $L_{\Lambda(k)}$ denote the fundamental cardinal $L$-spline with respect to the differential operator $M_{\Lambda(k)}$. Now using the Fourier-Laplace series of $f$ we want to define a fundamental polyspline $L_{f}$ by

$$
L_{f}(r \theta):=\sum_{k=0}^{\infty} \sum_{l=1}^{a_{k}} f_{k, l} \cdot L_{\Lambda(k)}(\log r) \cdot Y_{k, l}(\theta) .
$$

The series converges absolutely and uniformly since by (37) and (42) we have the estimate:

$$
\left|L_{f}(r \theta)\right| \leq M e^{-\varepsilon|\log r|} \sum_{k=0}^{\infty} \sum_{l=1}^{a_{k}}\left|f_{k, l}\right|\left|Y_{k, l}(\theta)\right| \leq K\|f\|_{\frac{n}{2}-1} .
$$

Furthermore $L_{f}$ is polyharmonic on each annulus $A\left(e^{j}, e^{j+1}\right)$ since each summand $L_{\Lambda(k)}(\log r) \cdot Y_{k, l}(\theta)$ is according to the results in Section 2 polyharmonic of order $p$ and the uniform limit of such functions is again polyharmonic of order $p$.

Since $L_{\Lambda(k)}(0)=1$ and $L_{\Lambda(k)}(j)=0$, for all $j \in \mathbb{Z}, j \neq 0$, we conclude that $L_{f}$ interpolates the given data $f$, i.e. (43) holds.

We want to prove that the partial derivatives of $\theta \rightarrow L_{f}(r \theta)$ and $r \rightarrow L_{f}(r \theta)$ exist up to the order $2(p-1)$. It suffices to prove the uniform convergence of the series

$$
\sum_{k=0}^{\infty} \sum_{l=1}^{a_{k}} f_{k, l} \cdot \frac{d^{m}}{d r^{m}} L_{\Lambda(k)}(\log r) \cdot D^{\alpha} Y_{k, l}(\theta) ;
$$

for $m+|\alpha| \leq 2 p-2$. By formula (15), and Theorem 12, there exist constants $C>0$ and $\varepsilon>0$ and $k_{0} \in \mathbb{N}$ such that for all $k \geq k_{0}$ holds

$$
\left|\frac{d^{m}}{d r^{m}} L_{\Lambda(k)}(\log r)\right| \leq C k^{m} e^{-\varepsilon|\log r|}
$$


By [22], or [21], there exists a constant $K>0$ independent of $k$ such that for all $Y_{k} \in \mathcal{H}_{k}$, and for all $\alpha \in \mathbb{N}_{0}$ with $|\alpha| \leq N:=2(p-1)-m$, the following estimate holds

$$
\left|D^{\alpha} Y_{k}(\theta)\right| \leq K \cdot k^{(n / 2)-1+N}\left\|Y_{k}(\theta)\right\|_{L_{2}\left(\mathbb{S}^{n-1}\right)} .
$$

Applying the last inequality to $Y_{k, l}(\theta)$ (note that $\left\|Y_{k, l}(\theta)\right\|_{L_{2}\left(\mathbb{S}^{n-1}\right)}=1$ ) we obtain that for all $\alpha \in \mathbb{N}_{0}^{n-1}$ with $|\alpha| \leq N:=2(p-1)-m$

$$
\begin{aligned}
& \sum_{k=0}^{\infty} \sum_{l=1}^{a_{k}}\left|f_{k, l} \cdot \frac{d^{m}}{d r^{m}} L_{\Lambda(k)}(\log r) \cdot D^{\alpha} Y_{k, l}(\theta)\right| \\
\leq & C K e^{-\varepsilon|\log r|} \sum_{k=0}^{\infty} \sum_{l=1}^{a_{k}}\left|f_{k, l}\right| \cdot k^{m} \cdot k^{(n / 2)-1+N} \\
= & C K e^{-\varepsilon|\log r|} \sum_{k=0}^{\infty} \sum_{l=1}^{a_{k}}\left|f_{k, l}\right| \cdot k^{2(p-1)} \cdot k^{(n / 2)-1} .
\end{aligned}
$$

Since $\|f\|_{s_{p, n}}<\infty$ we conclude that $L_{f}(r \theta)$ is differentiable up to the order $2(p-1)$ and $(45)$ holds.

\subsection{Construction of interpolation polysplines}

Now let us construct interpolation polysplines. Assume that $d_{j}$ are data functions defined on the spheres $e^{j} \mathbb{S}^{n-1}$. Then we put $f_{j}(\theta):=d_{j}\left(e^{j} \theta\right)$, consequently $f_{j}$ is a function on the sphere $\mathbb{S}^{n-1}$.

Theorem 17 Let $\gamma \geq 0$ and $s=s_{p, n}=2(p-1)+(n / 2)-1$ and $f_{j} \in$ $H^{s, 1}\left(\mathbb{S}^{n-1}\right)$ for $j \in \mathbb{Z}$. Suppose that there exists a constant $C>0$ such that the inequality

$$
\left\|f_{j}\right\|_{s} \leq C|j|^{\gamma}=C\left|\log e^{j}\right|^{\gamma}
$$

holds for all $j \in \mathbb{Z}$. Then there exists a polyspline $S: \mathbb{R}^{n} \backslash\{0\} \rightarrow \mathbb{R}$ of order $p$ such that

$$
S\left(e^{j} \theta\right)=f_{j}(\theta)=d_{j}\left(e^{j} \theta\right) \quad \text { for all } \theta \in \mathbb{S}^{n-1}
$$

holds for each $j \in \mathbb{Z}$, and there exists a constant $D>0$ such that for all $\theta \in \mathbb{S}^{n-1}$ and all $r>0$

$$
|S(r \theta)| \leq D|\log r|^{\gamma}
$$


PROOF. The following well known fact can be found e.g. in Schoenberg [18]: Let $\gamma \geq 0$ and $\varepsilon>0$. Then there exists $D(\varepsilon, \gamma)>0$ and $R_{0}>0$ such that for all $x \in \mathbb{R}$ with $|x| \geq R_{0}$ the following inequality holds:

$$
\sum_{j=-\infty}^{\infty}|j|^{\gamma} e^{-\varepsilon|x-j|} \leq D(\varepsilon, \gamma)|x|^{\gamma} .
$$

For each $f_{j}$ we can define a fundamental polyspline $L_{f_{j}}$ as in Theorem 16. We define the interpolation polyspline by putting

$$
S(x):=\sum_{j=-\infty}^{\infty} L_{f_{j}}\left(x e^{-j}\right) .
$$

The estimate (45) yields $\left|L_{f_{j}}\left(x e^{-j}\right)\right| \leq M e^{-\varepsilon|\log | x e^{-j}}\|\| f_{j} \|_{s}$, hence by (49) and (50) it follows

$$
|S(x)| \leq \sum_{j=-\infty}^{\infty} M C e^{-\varepsilon|\log | x e^{-j}||}|j|^{\gamma} \leq C M D(\varepsilon, \gamma)|\log | x||^{\gamma} .
$$

This shows that $S$ is well-defined and since the convergence is locally uniform it is clear that $S$ is continuous on $\mathbb{R}^{n} \backslash\{0\}$ and polyharmonic on the open annuli $A\left(e^{j}, e^{j+1}\right)$ for $j \in \mathbb{Z}$.

The differentiability of $S$ up to order $2(p-1)$ follows from similar estimates using inequality (45). Then

$$
\sum_{j=-\infty}^{\infty}\left|\frac{d^{m}}{d r^{m}} D^{\alpha} L_{f_{j}}\left(r \theta e^{-j}\right)\right| \leq \sum_{j=-\infty}^{\infty} M e^{-\varepsilon\left|\log r e^{j}\right|}\left\|f_{j}\right\|_{s} .
$$

This ends the proof.

\section{$7 \quad$ Uniqueness of interpolation polysplines}

In this Section we will prove uniqueness of interpolation polysplines.

Theorem 18 Let $\gamma \geq 0$. Suppose $S_{1}, S_{2}: \mathbb{R}^{n} \backslash\{0\} \rightarrow \mathbb{C}$ be polysplines of order $p$ such that

$$
\left|S_{i}(r \theta)\right| \leq C\left(|\log r|^{\gamma}\right)
$$


for $i=1,2$. If $S_{1}\left(e^{j} \theta\right)=S_{2}\left(e^{j} \theta\right)$ for all $j \in \mathbb{Z}$ and for all $\theta \in \mathbb{S}^{n-1}$ then $S_{1} \equiv S_{2}$.

PROOF. Let us put $S:=S_{1}-S_{2}$. Let $S_{k, l}(\log r)$, with $v=\log r$, be the Fourier-Laplace coefficients of $S$ as defined in (6). According to Theorem 2, $S_{k, l}(v)$ are cardinal $L$-splines with respect to the linear differential operator $M_{\Lambda(k)}$ and clearly $S_{k, l}(j)=0$ for all $j \in \mathbb{Z}$. Further, by the assumption of the Theorem we see that for all $v \in \mathbb{R}$ inequality

$$
\left|S_{k, l}(v)\right| \leq \int_{\mathbb{S}^{n-1}}\left|S\left(e^{v} \theta\right) Y_{k, l}(\theta)\right| d \theta \leq C_{k, l}\left|\log e^{v}\right|^{\gamma}=C_{k, l}|v|^{\gamma}
$$

holds with some constants $C_{k, l}>0$. Hence $S_{k, l}$ is a cardinal $L$-spline of polynomial growth. By the uniqueness for interpolation cardinal $L$-splines (see [16, p. 204] applied for $\alpha=0$ ) we infer that $S_{k, l} \equiv 0$. This implies $S \equiv 0$ and finishes the proof.

\section{References}

[1] Adams, R., Sobolev Spaces, Academic Press, New York, 1975.

[2] Axler, S., Bourdon, P., Ramey, W., Harmonic Function Theory, Springer-Verlag, New York, 1992.

[3] Baker, A., Transcendental Number Theory, Cambridge University Press, Cambridge, 1975.

[4] Bejancu, A., Kounchev, O., Render, H., Cardinal Interpolation with biharmonic polysplines on strips, Proceedings of the Fifth International Conference on "Curves and Surfaces", Saint-Malo, 27.6.-3.7.2002, Nashboro Press 2003.

[5] Chui, Ch., An introduction to wavelets, Academic Press, Boston, 1992.

[6] Conway, J.B., Functions of one complex variable, Springer Verlag, 2nd ed., New York, 1978.

[7] Dyn, N., Ron, A., Recurrence relations for Tchebycheffian B-splines, Journal d'Analyse Mathem., 51 (1988), pp. 118-138.

[8] Kalf, H., On the expansion of a function in terms of spherical harmonics in arbitrary dimensions, Bull. Belg. Math. Soc. 2 (1995) 361-380.

[9] Kounchev, O.I., Minimizing the integral of the Laplacian of a function squared with prescribed values on interior boundaries - theory of polysplines, Trans. Amer. Math. Soc. 350 (1998), 2105-2128. 
[10] Kounchev, O.I., Multivariate Polysplines. Applications to Numerical and Wavelet Analysis, Academic Press, London-San Diego, 2001.

[11] Kounchev, O., Render, H., Symmetry properties for cardinal L-splines with applications to polysplines, In: K. Kopotun, T. Lyche and M. Neamtu (eds.), Trends in Approximation theory, Vanderbilt University Press, Nashville, TN, 2001, pp. 191-202.

[12] Kounchev, O., Render, H., Wavelet Analysis of cardinal L-splines and Construction of multivariate Prewavelets, In: Chui, Charles (ed.) et al., Approximation Theory X. Wavelets, splines, and Applications, Vanderbilt University Press, Nashville, TN, 2002, pp. 333-353.

[13] Kounchev, O., Render, H., The Approximation order of Polysplines, Proc. Amer. Math. Soc. 132 (2004), 455-461.

[14] Kounchev, O., Render, H., Polyharmonic splines on grids $\mathbb{Z} \times a \mathbb{Z}^{n}$ and their limits for $a \rightarrow 0$, to appear in Mathematics of Computation.

[15] Mahler, K., Lectures on transcendental numbers, Lecture Notes in Mathematics, 546, Springer-Verlag, 1976.

[16] Micchelli, Ch., Oscillation matrices and cardinal spline interpolation, In: Studies in Spline Functions and Approximation Theory, Eds. S. Karlin et al., Academic Press, NY, 1976, pp.163-202.

[17] Micchelli, Ch., Cardinal L-splines, In: Studies in Spline Functions and Approximation Theory, Eds. S. Karlin et al., Academic Press, NY, 1976, pp. 203-250.

[18] Schoenberg, I. J., Cardinal interpolation and spline functions, Jour. Approx. Theory, 2 (1969), pp. 167-206.

[19] Schoenberg, I. J., Cardinal Spline Interpolation, SIAM, Philadelphia, Pennsylvania, 1973.

[20] Schoenberg, I.J., On Micchelli's theory of cardinal $L$-splines, In: Studies in Spline Functions and Approximation Theory, Eds. S. Karlin et al., Academic Press, NY, 1976, pp. 251-276.

[21] Seeley, R., Spherical harmonics, Amer. Math. Monthly, 73 (1966), 115-121.

[22] Sobolev, S.L., Cubature Formulas and Modern Analysis: An introduction, Gordon and Breach Science Publishers, Montreux, 1992.

[23] Stein, E.M., Weiss, G., Introduction to Fourier Analysis on Euclidean Spaces, Princeton University Press, Princeton, 1971. 